\title{
Irregular fibers of complex polynomials in two variables
}

\author{
Arnaud BoDIN \\ Laboratoire Paul Painlevé \\ UFR de Mathématiques Université Lille I \\ 59655 Villeneuve d'Ascq Cédex \\ FRANCE \\ Arnaud.Bodin@math.univ-lille1.fr
}

Recibido: 8 de Julio de 2002

Aceptado: 7 de Febrero de 2003

\begin{abstract}
For a complex polynomial in two variables we study the morphism induced in homology by the embedding of an irregular fiber in a regular neighborhood of it. We give necessary and sufficient conditions for this morphism to be injective, surjective. Particularly this morphism is an isomorphism if and only if the corresponding irregular value is regular at infinity. We apply these results to the study of vanishing and invariant cycles.
\end{abstract}

Key words: irregulars fibers, tube, vanishing cycles, invariant cycles, resolution of singularities

2000 Mathematics Subject Classification: 32S20, 32S45, 32S30

\section{Introduction}

Let $f: \mathbb{C}^{n} \longrightarrow \mathbb{C}$ be a polynomial. The bifurcation set $\mathcal{B}$ for $f$ is the minimal set of points of $\mathbb{C}$ such that $f: \mathbb{C}^{n} \backslash f^{-1}(\mathcal{B}) \longrightarrow \mathbb{C} \backslash \mathcal{B}$ is a locally trivial fibration. For $c \in \mathbb{C}$, we denote the fiber $f^{-1}(c)$ by $F_{c}$. The fiber $F_{c}$ is irregular if $c$ is in $\mathcal{B}$. If $s \notin \mathcal{B}$, then $F_{s}$ is a generic fiber and is denoted by $F_{\text {gen }}$. The tube $T_{c}$ for the value $c$ is a neighborhood $f^{-1}\left(D_{\varepsilon}^{2}(c)\right)$ of the fiber $F_{c}$, where $D_{\varepsilon}^{2}(c)$ stands for a 2-disk in $\mathbb{C}$, centered at $c$, of radius $\varepsilon \ll 1$. We assume that affine critical singularities are isolated. The value $c$ is regular at infinity if there exists a sufficiently large compact set $K$ of $\mathbb{C}^{n}$ such that the restriction of $f, f: T_{c} \backslash K \longrightarrow D_{\varepsilon}^{2}(c)$ is a locally trivial fibration. 
Set $n=2$. Let $j_{c}: H_{1}\left(F_{c}\right) \longrightarrow H_{1}\left(T_{c}\right)$ be the morphism induced by the inclusion of $F_{c}$ in $T_{c}$. The first part of this work is the study of this morphism. Let $G_{c}$ the dual graph of $F_{c}=f^{-1}(c)$, and $\bar{G}_{c}$ the dual graph of a compactification of the fiber $F_{c}$ obtained by a resolution at infinity of $f$. The value $c$ is acyclic if the dual graph $G_{c}$ and some dual graphs $G_{c, P}$ obtained by compactification have the same number of cycles (see the full definition later). This is a combinatoric condition, for example if the fiber $F_{c}$ is connected then $c$ is acyclic if and only if $H_{1}\left(G_{c}\right)$ is isomorphic to $H_{1}\left(\bar{G}_{c}\right)$. Finally we define $j_{\infty}: H_{1}\left(F_{c} \backslash K\right) \longrightarrow H_{1}\left(T_{c} \backslash K\right)$ induced by inclusion (this map is independent of the sufficiently large set $K$ ).

\section{Theorem.}

(A) $j_{c}$ is injective if and only if $F_{c}$ is connected and $c$ is acyclic.

(B) $j_{c}$ is surjective if and only if $j_{\infty}$ is surjective and $c$ is acyclic.

(C) $j_{c}$ is an isomorphism if and only if $c$ is a regular value at infinity.

It should be noticed that the fiber $F_{c}$ is not supposed to be smooth. In fact we have a stronger result for the part (A) because the rank of the kernel of $j_{c}$ is:

$$
\operatorname{rk} \operatorname{Ker} j_{c}=n\left(F_{c}\right)-1+\operatorname{rk} H_{1}\left(\bar{G}_{c}\right)-\operatorname{rk} H_{1}\left(G_{c}\right)
$$

where $n\left(F_{c}\right)$ is the number of connected components of $F_{c}$. The acyclicity condition and the surjectivity of $j_{\infty}$ can be checked from the dual graphs of resolution. E. Artal-Bartolo, Pi. Cassou-Noguès and A. Dimca have proved the part (C) in [1] for polynomials with a connected fiber $F_{c}$. For a non-connected fiber $j_{c}$ is not injective (part (A)), and we could have used the result of [1] to prove part (C) but we will give a complete proof.

We apply these results to the study of neighborhoods of irregular fibers. Set $n \geqslant 2$. Let $F_{c}^{\circ}$ be the smooth part of $F_{c}: F_{c}^{\circ}$ is obtained by intersecting $F_{c}$ with a large $2 n$-ball and cutting out a small neighborhood of the (isolated) singularities. Then $F_{c}^{\circ}$ can be embedded in $F_{g e n}$. We study the following commutative diagram that links the three elements $F_{c}^{\circ}, F_{g e n}$, and $T_{c}$ :

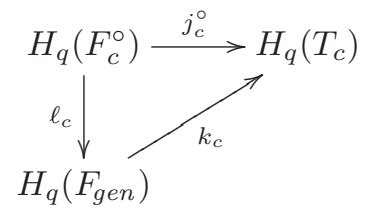

where $\ell_{c}$ is the morphism induced in integral homology by the embedding; $j_{c}^{\circ}$ and $k_{c}$ are induced by inclusions. The morphism $k_{c}$ is well-known and $V_{q}(c)=\operatorname{Ker} k_{c}$ are the vanishing cycles for the value $c$. Let $h_{c}$ be the monodromy induced on $H_{q}\left(F_{g e n}\right)$ by a 
small circle around the value $c$. Then we prove that the image of $\ell_{c}$ are the invariant cycles by $h_{c}$ :

$$
\operatorname{Ker}\left(h_{c}-\mathrm{id}\right)=\ell_{c}\left(H_{q}\left(F_{c}^{\circ}\right)\right) .
$$

This formula for the case $n=2$ has been obtained by F. Michel and C. Weber in [6].

Finally we give a description of vanishing cycles with respect to the eigenvalues of $h_{c}$ for homology with complex coefficients. For $\lambda \neq 1$ and $p$ a large integer the characteristic space $E_{\lambda}=\operatorname{Ker}\left(h_{c}-\lambda \mathrm{id}\right)^{p}$ is generated by vanishing cycles for the value $c$. For $\lambda=1$ the situation is different. If $K_{q}(c)=V_{q}(c) \cap \operatorname{Ker}\left(h_{c}-\right.$ id $)$ are invariant and vanishing cycles we have

$$
K_{q}(c)=\ell_{c}\left(\operatorname{Ker} j_{c}^{\circ}\right) .
$$

For $n=2$ we have $E_{1}=\operatorname{Ker}\left(h_{c}-\mathrm{id}\right)^{2}$ and we prove that $\operatorname{Ker}\left(h_{c}-\mathrm{id}\right)^{2} \cap V_{1}(c)=$ $\operatorname{Ker}\left(h_{c}-\mathrm{id}\right) \cap V_{1}(c)=: K_{1}(c)$ with the formula

$$
\operatorname{rk} K_{1}(c)=r\left(F_{c}\right)-1+\operatorname{rk} H_{1}\left(\bar{G}_{c}\right),
$$

where $r\left(F_{c}\right)$ is the number of irreducible components of $F_{c}$.

The study of vanishing cycles is motivated by the following result of [3]: the monodromy $h_{\infty}: H_{1}\left(F_{g e n}\right) \longrightarrow H_{1}\left(F_{\text {gen }}\right)$ induced by a large circle around the set $\mathcal{B}$ and Broughton's decomposition $H_{1}\left(F_{\text {gen }}\right)=\bigoplus_{c \in \mathcal{B}} V_{1}(c)$ determine the monodromy representation $\pi_{1}(\mathbb{C} \backslash \mathcal{B}) \longrightarrow \operatorname{Aut} H_{1}\left(F_{\text {gen }}\right)$. The formula for $\operatorname{rk} K_{1}(c)$ enables to describe vanishing cycles with respect to a decomposition of the homology of the generic fiber given by the resolution of singularities.

\section{Irregular fibers and tubes}

\subsection{Bifurcation set}

We can describe the bifurcation set $\mathcal{B}$ as follows: let Sing $=\left\{z \in \mathbb{C}^{n} \mid \operatorname{grad}_{f}(z)=0\right\}$ be the set of affine critical points and let $\mathcal{B}_{a f f}=f$ (Sing) be the set of affine critical values. The set $\mathcal{B}_{\text {aff }}$ is a subset of $\mathcal{B}$. The value $c \in \mathbb{C}$ is regular at infinity if there exist a disk $D$ centered at $c$ and a compact set $K$ of $\mathbb{C}^{n}$ with a locally trivial fibration $f: f^{-1}(D) \backslash K \longrightarrow D$. The non-regular values at infinity are the critical values at infinity and are collected in $\mathcal{B}_{\infty}$. The finite set $\mathcal{B}$ of critical values is now:

$$
\mathcal{B}=\mathcal{B}_{\text {aff }} \cup \mathcal{B}_{\infty} .
$$

In this article we always assume that affine singularities are isolated, that is to say that Sing is an isolated set in $\mathbb{C}^{n}$. For $n=2$ this hypothesis implies that the generic fiber is a connected set. 


\subsection{Preliminaries}

In this paragraph $n=2$. The inclusion of $F_{c}$ in $T_{c}$ induces a morphism $j_{c}: H_{1}\left(F_{c}\right) \longrightarrow$ $H_{1}\left(T_{c}\right)$. We firstly recall notations and results from [1].

Let denote $F_{\text {aff }}=F_{c} \cap B_{R}^{4}(R \gg 1)$ and $F_{\infty}=\overline{F_{c} \backslash F_{a f f}}$, thus $F_{\text {aff }} \cap F_{\infty}=K_{c}=$ $f^{-1}(c) \cap S_{R}^{3}$ is the link at infinity for the value $c$. Similarly $T_{a f f}=T_{c} \cap B_{R}^{4}$ and $T_{\infty}=$ $\overline{T_{c} \backslash T_{\text {aff }}}$. We denote $j_{\infty}: H_{1}\left(F_{\infty}\right) \longrightarrow H_{1}\left(T_{\infty}\right)$ the morphism induced by inclusion. The inclusion $F_{\text {aff }} \subset T_{\text {aff }}$ is a homotopy equivalence so $j_{\text {aff }}: H_{1}\left(F_{\text {aff }}\right) \longrightarrow H_{1}\left(T_{a f f}\right)$ is an isomorphism. $H_{1}\left(F_{\text {aff }} \cap F_{\infty}\right)$ and $H_{1}\left(T_{a f f} \cap T_{\infty}\right)$ are isomorphic.

The Mayer-Vietoris exact sequences for the decompositions $F_{c}=F_{a f f} \cup F_{\infty}$ and $T_{c}=T_{a f f} \cup T_{\infty}$ give the commutative diagram $(\mathcal{D})$ :

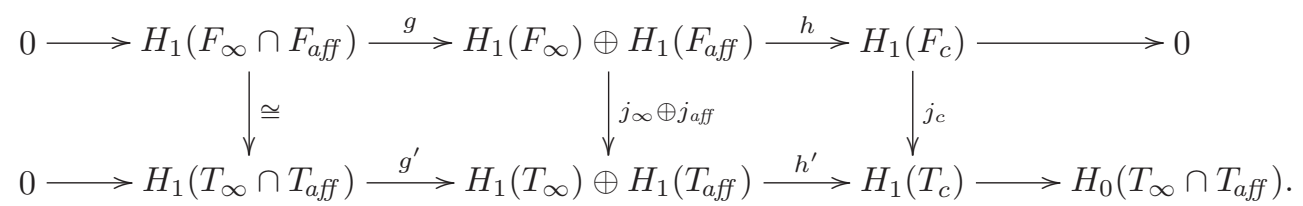

The 0 at the upper-right corner is provided by the injectivity of $H_{0}\left(F_{\infty} \cap F_{\text {aff }}\right) \longrightarrow$ $H_{0}\left(F_{\infty}\right)$ hence $H_{0}\left(F_{\infty} \cap F_{a f f}\right) \longrightarrow H_{0}\left(F_{\infty}\right) \oplus H_{0}\left(F_{a f f}\right)$ is injective (notice that $F_{c}$ need not to be a connected set).

\subsection{Resolution of singularities}

To compactify the situation, for $n=2$, we need the resolution of singularities at infinity [5]:

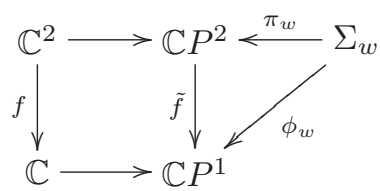

Here $\tilde{f}$ is a rational map coming from the homogenisation of $f$ and is not defined at some points of the line at infinity $L_{\infty}$ of $\mathbb{C} P^{2} ; \pi$ is the minimal blow-up at these points in order to obtain a well-defined morphism $\phi_{w}: \Sigma_{w} \longrightarrow \mathbb{C} P^{1}$ : this is the weak resolution. We denote $\phi_{w}^{-1}(\infty)$ by $D_{\infty}$, and let $D_{\text {dic }}$ be the set of components $D$ of $\pi_{w}^{-1}\left(L_{\infty}\right)$ that verify $\phi_{w}(D)=\mathbb{C} P^{1}$. Such a $D$ is a dicritical component. The degree of a dicritical component $D$ is the degree of the branched covering $\phi_{w}: D \longrightarrow \mathbb{C} P^{1}$. For the weak resolution the divisor $\phi_{w}^{-1}(c) \cap \pi_{w}^{-1}\left(L_{\infty}\right), c \in \mathbb{C}$, is a union of bamboos (possibly empty). A bamboo is a divisor whose dual graph is a linear tree. The set $\mathcal{B}_{\infty}$ is the set of values of $\phi_{w}$ on non-empty bamboos union the set of critical values of the restriction of $\phi_{w}$ to the dicritical components. 
We can blow-up more points to obtain the total resolution, $\phi_{t}: \Sigma_{t} \longrightarrow \mathbb{C} P^{1}$, such that all fibers of $\phi_{t}$ are normal crossing divisors that intersect the dicritical components transversally; moreover we solve affine singularities. Then $D_{\infty}=\phi_{t}^{-1}(\infty)$ is the same as above and for $c \in \mathcal{B}$ we denote $D_{c}$ the divisor $\phi_{t}^{-1}(c)$.

The dual graph $\bar{G}_{c}$ of $D_{c}$ is obtained as follows: one vertex for each irreducible component of $D_{c}$ and one edge between two vertices for one intersection of the corresponding components. A similar construction is done for $D_{\infty}$, we know that $\bar{G}_{\infty}$ is a tree [5]. The multiplicity of a component is the multiplicity of $\phi_{t}$ on this component.

\subsection{Study of $j_{\infty}$}

See [1]. Let $\phi$ be the weak resolution map for $f$. Let denote by $\operatorname{Dic}_{c}$ the set of points $P$ in the dicritical components, such that $\phi(P)=c$. To each $P \in \mathrm{Dic}_{c}$ is associated one, and only one, connected component $T_{P}$ of $T_{\infty} ; T_{P}$ is called the place at infinity for $P$. We have $T_{\infty}=\bigsqcup_{P \in \text { Dic }_{c}} T_{P}$ and we set $F_{P}=T_{P} \cap F_{\infty}=T_{P} \cap F_{c}$ and $K_{P}=\partial F_{P}$, finally $n\left(F_{P}\right)$ denotes the number of connected components of $F_{P}$. Let $\bar{F}_{P}$ be the strict transform of $c$ by $\phi$, intersected with $T_{P}$. The map $j_{\infty}$ is the direct sum of the maps $j_{P}: H_{1}\left(F_{P}\right) \longrightarrow H_{1}\left(T_{P}\right)$. Let $\mathfrak{m}_{P}$ be the intersection multiplicity of $\bar{F}_{P}$ with the divisor $\pi_{w}^{*}\left(L_{\infty}\right)$ at $P$.

Case of $\boldsymbol{P} \in \overline{\boldsymbol{F}}_{\boldsymbol{P}}$. The group $H_{1}\left(T_{P}\right)$ is isomorphic to $\mathbb{Z}$ and is generated by $\left[M_{P}\right]$, $M_{P}$ being the boundary of a small disk with transversal intersection with the dicritical component. Moreover if $F_{P}=\coprod_{i=1}^{n\left(F_{P}\right)} F_{P}^{i}$ then $j_{P}\left(\left[F_{P}^{i}\right]\right)=j_{P}\left(\left[K_{P}^{i}\right]\right)=\mathfrak{m}_{P}^{i}\left[M_{P}\right]$.

Case of $\boldsymbol{P}$ being in a bamboo. The group $H_{1}\left(T_{P}\right)$ is also isomorphic to $\mathbb{Z}$ and is generated by $\left[M_{P}\right], M_{P}$ being the boundary of a small disk, with transversal intersection with the last component of the bamboo. Then $j_{P}\left[F_{P}^{i}\right]=j_{P}\left[K_{P}^{i}\right]=\mathfrak{m}_{P}^{i} \cdot \ell_{i}\left[M_{P}\right]$. The integer $\ell_{i}$ only depends of the position where $F_{P}^{i}$ intersects the bamboo, moreover $\ell_{i} \geqslant 1$ and $\ell_{i}=1$ if and only if $F_{P}^{i}$ intersects the bamboo at the last component. For a computation of $\ell_{i}$ with the help of continuous fraction associated to the bamboos, refer to [1].

As a consequence $j_{P}$ is injective if and only if $n\left(F_{P}\right)=1$ and $j_{\infty}$ is injective if and only if $n\left(F_{P}\right)=1$ for all $P$ in $\mathrm{Dic}_{c}$. In fact the rank of the kernel of $j_{\infty}$ is the sum of the ranks of the kernels of $j_{P}$ then we deduce:

$$
\text { rk ker } j_{\infty}=\sum_{P \in \text { Dic }_{c}}\left(n\left(F_{P}\right)-1\right) .
$$

Finally $j_{\infty}$ is surjective if and only if for all $P \in \operatorname{Dic}_{c}, j_{P}$ is surjective. 


\subsection{Acyclicity}

The value $c$ is acyclic if the morphism $\psi: H_{0}\left(T_{\infty} \cap T_{\text {aff }}\right) \longrightarrow H_{0}\left(T_{\infty}\right) \oplus H_{0}\left(T_{a f f}\right)$ given by the Mayer-Vietoris exact sequence is injective.

Let give some interpretations of the acyclicity condition.

(i) The injectivity of $\psi$ can be viewed as follows: two branches at infinity that intersect the same place at infinity have to be in different connected components of $F_{c}$.

(ii) Let $G_{c}$ be the dual graph of $F_{c}$ (one vertex for an irreducible component of $F_{c}$, two vertices are joined by an edge if the corresponding irreducible components have non-empty intersection, if a component has auto-intersection it provides a loop) and let $G_{c, P}$ be the graph obtained from $G_{c}$ by adding edges to vertices that correspond to the same place at infinity $T_{P}$. In other words $c$ is acyclic if and only if there is no new cycles in $G_{c, P}$, that is to say $H_{1}\left(G_{c}\right) \cong H_{1}\left(G_{c, P}\right)$ for all $P$ in $\mathrm{Dic}_{c}$.

(iii) Another interpretation is the following: $c$ is acyclic if and only if the morphism $h^{\prime}$ of the diagram $(\mathcal{D})$ is surjective. This can be proved with the exact sequence:

$$
\begin{gathered}
H_{1}\left(T_{\infty}\right) \oplus H_{1}\left(T_{a f f}\right) \stackrel{h^{\prime}}{\longrightarrow} H_{1}\left(T_{c}\right) \stackrel{\varphi}{\longrightarrow} H_{0}\left(T_{\infty} \cap T_{a f f}\right) \stackrel{\psi}{\longrightarrow} \\
\stackrel{\psi}{\longrightarrow} H_{0}\left(T_{\infty}\right) \oplus H_{0}\left(T_{a f f}\right) \longrightarrow H_{0}\left(T_{c}\right) \longrightarrow 0
\end{gathered}
$$

(iv) Let consider the above Mayer-Vietoris exact sequence in reduced homology, the morphism $\widetilde{\psi}: \widetilde{H}_{0}\left(T_{\infty} \cap T_{a f f}\right) \longrightarrow \widetilde{H}_{0}\left(T_{\infty}\right) \oplus \widetilde{H}_{0}\left(T_{a f f}\right)$ is surjective because $\widetilde{H}_{0}\left(T_{c}\right)=\{0\}$. Moreover $\widetilde{\psi}$ is injective if and only if $\psi$ is injective. As $\widetilde{\psi}$ is surjective, $\widetilde{\psi}$ is injective if and only if $\operatorname{rk} \widetilde{H}_{0}\left(T_{\infty} \cap T_{\text {aff }}\right)=\operatorname{rk} \widetilde{H}_{0}\left(T_{\infty}\right)+\operatorname{rk} \widetilde{H}_{0}\left(T_{a f f}\right)$, but $T_{\infty}$ has exactly $\# \mathrm{Dic}_{c}$ connected components (see paragraph 1.4 ). Then $c$ is acyclic if and only if

$$
\sum_{P \in \operatorname{Dic}_{c}} n\left(F_{P}\right)-1=\# \operatorname{Dic}_{c}-1+n\left(F_{c}\right)-1
$$

This implies the lemma:

Lemma 1.1. $j_{\infty}$ is injective $\Longleftrightarrow F_{c}$ is a connected set and $c$ is acyclic.

Proof. If $j_{\infty}$ is injective then $n\left(F_{P}\right)=1$ for all $P$ in $\operatorname{Dic}_{c}$, then $H_{0}\left(T_{\infty} \cap T_{a f f}\right) \cong$ $H_{0}\left(T_{\infty}\right)$ and $\psi$ is injective, hence $c$ is acyclic and from equality $(\star)$, we have $n\left(F_{c}\right)=1$ i.e. $F_{c}$ is a connected set. Conversely, if $c$ is acyclic and $n\left(F_{c}\right)=1$ then equality $(\star)$ gives $n\left(F_{P}\right)=1$ for all $P$ in $\operatorname{Dic}_{c}$, thus $j_{\infty}$ is injective. 
Let us define a stronger notion of acyclicity. Let $\bar{G}_{c}$ be the dual graph of $\phi^{-1}(c)$. The graph $\bar{G}_{c}$ can be obtained from $G_{c}$ by adding edges between vertices that belong to the same place at infinity for all $P$ in $\mathrm{Dic}_{c}$. The value $c$ is strongly acyclic if $H_{1}\left(\bar{G}_{c}\right) \cong H_{1}\left(G_{c}\right)$. Strong acyclicity implies acyclicity, but the converse can be false. However if $F_{c}$ is a connected set (that is to say $G_{c}$ is a connected graph) then both conditions are equivalent. This is implicitly expressed in the next lemma, which is just a result involving graphs.

Lemma 1.2. $\operatorname{rk} H_{1}\left(\bar{G}_{c}\right)-\operatorname{rk} H_{1}\left(G_{c}\right)=\sum_{P \in \mathrm{Dic}_{c}}\left(n\left(F_{P}\right)-1\right)-\left(n\left(F_{c}\right)-1\right)$.

\subsection{Surjectivity}

Part (B). $j_{c}$ surjective $\Longleftrightarrow j_{\infty}$ surjective and c acyclic.

Proof. Let us suppose that $j_{c}$ is surjective then a version of the five lemma applied to diagram $(\mathcal{D})$ proves that $j_{\infty}$ is surjective. As $j_{c}$ and $j_{\infty}$ are surjective, diagram $(\mathcal{D})$ implies that $h^{\prime}: H_{1}\left(T_{\infty}\right) \oplus H_{1}\left(T_{\text {aff }}\right) \longrightarrow H_{1}\left(T_{c}\right)$ is surjective, that means that $c$ is acyclic.

Conversely if $j_{\infty}$ is surjective and $c$ is acyclic then $h^{\prime}$ is surjective and diagram $(\mathcal{D})$ implies that $j_{c}$ is surjective.

\subsection{Injectivity}

Part (A). $j_{c}$ is injective $\Longleftrightarrow F_{c}$ is a connected set and $c$ is acyclic.

It follows from lemma 1.1 and from the next lemma.

Lemma 1.3. $j_{c}$ injective $\Longleftrightarrow j_{\infty}$ injective.

Moreover the rank of the kernel is:

$$
\text { rk ker } j_{c}=\operatorname{rkker} j_{\infty}=\sum_{P \in \operatorname{Dic}_{c}}\left(n\left(F_{P}\right)-1\right)=n\left(F_{c}\right)-1+\operatorname{rk} H_{1}\left(\bar{G}_{c}\right)-\operatorname{rk} H_{1}\left(G_{c}\right) .
$$

Proof. The first part of this lemma can be proved by a version of the five lemma. However we shall only prove the equality of the ranks of $\operatorname{ker} j_{c}$ and $\operatorname{ker} j_{\infty}$. It will imply the lemma because we already know that rk ker $j_{\infty}=\sum_{P \in \text { Dic }_{c}}\left(n\left(F_{P}\right)-1\right)$ (see paragraph 1.4) and from lemma 1.2 we then have $\operatorname{rk} \operatorname{ker} j_{\infty}=n\left(F_{c}\right)-1+\operatorname{rk} H_{1}\left(\bar{G}_{c}\right)-$ rk $H_{1}\left(G_{c}\right)$.

The study of the morphism $j_{c}: H_{1}\left(F_{c}\right) \longrightarrow H_{1}\left(T_{c}\right)$ is equivalent to the study of the morphism $H_{1}\left(T_{\text {aff }}\right) \longrightarrow H_{1}\left(T_{c}\right)$ induced by inclusion that, by abuse, will also be denoted by $j_{c}$. To see this, it suffices to remark that $F_{c}$ is obtained from $F_{a f f}=$ $F_{c} \cap B_{R}^{4}$ by gluing $F_{c} \cap S_{R}^{3} \times\left[0,+\infty\left[\right.\right.$ to its boundary $F_{c} \cap S_{R}^{3}$. We then have two homotopy equivalences $F_{c} \supset F_{c} \cap T_{a f f} \subset T_{a f f}$. Then the morphism $H_{1}\left(F_{a f f}\right)$ 
$H_{1}\left(F_{c}\right)$ induced by inclusion is an isomorphism; finally $j_{\text {aff }}: H_{1}\left(F_{\text {aff }}\right) \longrightarrow H_{1}\left(T_{\text {aff }}\right)$ is also an isomorphism. The long exact sequence for the pair $\left(T_{c}, T_{a f f}\right)$ is:

$$
H_{2}\left(T_{c}\right) \longrightarrow H_{2}\left(T_{c}, T_{\text {aff }}\right) \longrightarrow H_{1}\left(T_{\text {aff }}\right) \stackrel{j_{c}}{\longrightarrow} H_{1}\left(T_{c}\right)
$$

but $H_{2}\left(T_{c}\right)=0$ (see [1] for example) then the rank of ker $j_{c}$ is the rank of $H_{2}\left(T_{c}, T_{\text {aff }}\right)$.

On the other hand, the study of $j_{\infty}: H_{1}\left(F_{\infty}\right) \longrightarrow H_{1}\left(T_{\infty}\right)$ is the same as the study of $H_{1}\left(\partial T_{\infty}\right) \longrightarrow H_{1}\left(T_{\infty}\right)$ induced by inclusion (and denoted by $j_{\infty}$ ) because the morphisms $H_{1}\left(\partial F_{\infty}\right) \longrightarrow H_{1}\left(F_{\infty}\right)$ and $H_{1}\left(\partial F_{\infty}\right) \longrightarrow H_{1}\left(\partial T_{\infty}\right)$ induced by inclusions are isomorphisms. The long exact sequence for $\left(T_{\infty}, \partial T_{\infty}\right)$ is:

$$
H_{2}\left(T_{\infty}\right) \longrightarrow H_{2}\left(T_{\infty}, \partial T_{\infty}\right) \longrightarrow H_{1}\left(\partial T_{\infty}\right) \stackrel{j_{\infty}}{\longrightarrow} H_{1}\left(T_{\infty}\right) .
$$

As $H_{2}\left(T_{\infty}\right)=0$ (see [1]), then the rank of ker $j_{\infty}$ is equal to $\mathrm{rk} H_{2}\left(T_{\infty}, \partial T_{\infty}\right)$.

Finally the groups $H_{2}\left(T_{\infty}, \partial T_{\infty}\right)$ and $H_{2}\left(T_{c}, T_{a f f}\right)$ are isomorphic by excision, then the ranks of $\operatorname{ker} j_{c}$ and of $\operatorname{ker} j_{\infty}$ are equal. That completes the proof.

\subsection{Bijectivity}

Part (C). $j_{c}$ is an isomorphism $\Longleftrightarrow c \notin \mathcal{B}_{\infty}$

Proof. If $c \notin \mathcal{B}_{\infty}, F_{c}$ is homotopic to $F_{\text {aff }}$ and $T_{c}$ to $T_{\text {aff }}$ then the isomorphism $j_{\text {aff }}: H_{1}\left(F_{\text {aff }}\right) \longrightarrow H_{1}\left(T_{\text {aff }}\right)$ implies that $j_{c}$ is an isomorphism. Let suppose that $c$ is a critical value at infinity and that $j_{c}$ is injective. We have to prove that $j_{c}$ is not surjective. As $j_{c}$ is injective then by lemma $1.3, j_{\infty}$ is injective. By the part (B) it suffices to prove that $j_{\infty}$ is not surjective. Let $P$ be a point of Dic $_{c}$ that provides irregularity at infinity for the value $c$, then $n\left(F_{P}\right)=1$ because $j_{\infty}$ is injective. Let us prove that the morphism $j_{P}$ is not surjective. For the case of $P \in \bar{F}_{P}$, the intersection multiplicity $\mathfrak{m}_{P}$ is greater than 1 , then $j_{P}$ is not surjective. For the second case, in which $P$ belongs to a bamboo, we have $\mathfrak{m}_{P} . \ell_{i}>1$ except for the situation where only one strict transform intersects the bamboo at the last component (remember that $\ell_{i}=1$ if and only if we are at the last component of a bamboo). This is exactly the situation excluded by the lemma "bamboo extremity fiber" of [6]. Hence $j_{\infty}$ is not surjective and $j_{c}$ is not an isomorphism.

\subsection{Examples}

We apply the results to two classical examples.

Broughton polynomial. Let $f(x, y)=x(x y+1)$, then $\mathcal{B}_{\text {aff }}=\varnothing, \mathcal{B}=\mathcal{B}_{\infty}=\{0\}$. Then for $c \neq 0, j_{c}$ is an isomorphism. For the value $0, F_{0}$ contains two connected components $(x=0)$ and $(x y+1=0)$ so $G_{c}$ is composed of two vertices. The dual graph $\bar{G}_{0}$ contains also a vertex that corresponds to a component with multiplicity +1 of a bamboo. The value 0 is acyclic since $H_{1}\left(G_{0}\right) \cong H_{1}\left(\bar{G}_{0}\right)$. The fiber $F_{0}$ is not 
connected hence $j_{0}$ is not injective. As the new component of $\bar{G}_{0}$ is of multiplicity 1 the corresponding morphism $j_{\infty}$ is surjective, hence $j_{0}$ is surjective.

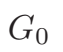

$G_{0}$

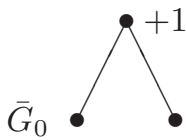

Briançon polynomial. Let $f(x, y)=y p^{3}+p^{2} s+a_{1} p s+a_{0} s$ with $s=x y+1, \quad p=$ $x(x y+1)+1, \quad a_{1}=-\frac{5}{3}, \quad a_{0}=-\frac{1}{3}$, see [2]. The bifurcation set is $\mathcal{B}=\mathcal{B}_{\infty}=\{0, c=$ $\left.-\frac{16}{9}\right\}$, moreover all fibers are smooth and irreducible. The value 0 is not acyclic then $j_{0}$ is neither injective nor surjective (but $j_{\infty}$ is surjective).
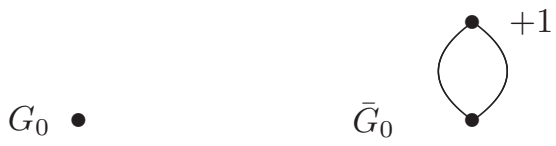

The value $c$ is acyclic, and $F_{c}$ is connected (since irreducible) then $j_{c}$ is injective. The morphism $j_{c}$ is not surjective: $j_{\infty}$ is not surjective because the compactification of $F_{c}$ does not intersect the bamboo at the last component.

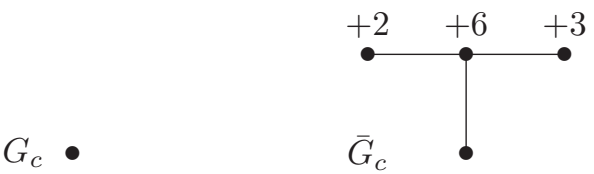

\section{Situation around an irregular fiber}

For $f: \mathbb{C}^{n} \longrightarrow \mathbb{C}$ we study the neighborood of an irregular fiber.

\subsection{Smooth part of $F_{c}$}

Let fix a value $c \in \mathbb{C}$ and let $B_{R}^{2 n}$ be a large closed ball $(R \gg 1)$. Let $B_{1}^{2 n}, \ldots, B_{p}^{2 n}$ be small open balls around the singular points (which are supposed to be isolated) of $F_{c}: F_{c} \cap$ Sing. We denote $B_{1}^{2 n} \cup \ldots \cup B_{p}^{2 n}$ by $B_{\cup}$. Then the smooth part of $F_{c}$ is

$$
F_{c}^{\circ}=F_{c} \cap\left(B_{R}^{2 n} \backslash B_{\cup}\right) .
$$

It is possible to embed $F_{c}^{\circ}$ in the generic fiber $F_{g e n}$ (see [6] and [8]). We now explain the construction of this embedding by W. Neumann and P. Norbury. As $F_{c}$ has transversal intersection with the balls of $B_{\cup}$ and with $B_{R}^{2 n}$, there exists a small 
disk $D_{\varepsilon}^{2}(c)$ such that, for all $s$ in this disk, $F_{s}$ has transversal intersection with these balls. According to Ehresmann fibration theorem, $f$ induces a locally trivial fibration

$$
f_{\mid}: f^{-1}\left(D_{\varepsilon}^{2}(c)\right) \cap\left(B_{R}^{2 n} \backslash B_{\cup}\right) \longrightarrow D_{\varepsilon}^{2}(c) .
$$

In fact, as $D_{\varepsilon}^{2}(c)$ is null homotopic, this fibration is trivial. Hence $F_{c}^{\circ} \times D_{\varepsilon}^{2}(c)$ is diffeomorphic to $f^{-1}\left(D_{\varepsilon}^{2}(c)\right) \cap\left(B_{R}^{2 n} \backslash B_{\cup}\right)$. That provides an embedding of $F_{c}^{\circ}$ in $F_{s}$ for all $s$ in $D_{\varepsilon}^{2}(c)$; and for such a $s$ with $s \neq c, F_{s}$ is a generic fiber. The morphism induced in homology by this embedding is denoted by $\ell_{c}$. Let $j_{c}^{\circ}$ be the morphism induced by the inclusion of $F_{c}^{\circ}$ in $T_{c}=f^{-1}\left(D_{\varepsilon}^{2}(c)\right)$. Similarly $k_{c}$ denotes the morphism induced by the inclusion of the generic fiber $F_{\text {gen }}=F_{s}$ (for $\left.s \in D_{\varepsilon}^{2}(c), s \neq c\right)$ in $T_{c}$. As all morphisms are induced by natural maps we have the lemma:

Lemma 2.1. The following diagram commutes:

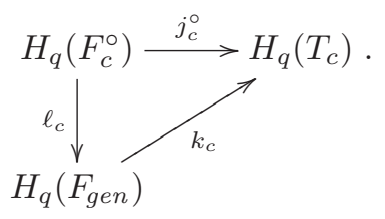

\subsection{Invariant cycles by $h_{c}$}

Invariant cycles by the monodromy $h_{c}$ can be recovered by the following property.

\section{Proposition 2.2.}

$$
\operatorname{Ker}\left(h_{c}-\mathrm{id}\right)=\ell_{c}\left(H_{q}\left(F_{c}^{\circ}\right)\right) .
$$

For $n=2$, there is a similar formula in [6], even for non-isolated singularities.

Proof. The proof uses a commutative diagram due to W. Neumann and P. Norbury [8]:

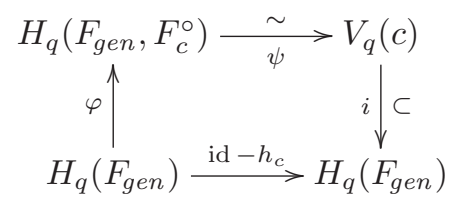

The morphism $i$ is the inclusion and $\psi$ is an isomorphism, so $\operatorname{Ker}\left(h_{c}-\right.$ id) equals $\operatorname{Ker} \varphi$. The long exact sequence for the pair $\left(F_{g e n}, F_{c}^{\circ}\right)$ is:

$$
\cdots \longrightarrow H_{q}\left(F_{c}^{\circ}\right) \stackrel{\ell_{c}}{\longrightarrow} H_{q}\left(F_{g e n}\right) \stackrel{\varphi}{\longrightarrow} H_{q}\left(F_{g e n}, F_{c}^{\circ}\right) \longrightarrow \cdots
$$

So $\operatorname{Im} \ell_{c}=\operatorname{Ker} \varphi=\operatorname{Ker}\left(h_{c}-\mathrm{id}\right)$. 
We are able to applicate this result to the calculus of the rank of $\operatorname{Ker}\left(h_{c}-\right.$ id) in $H_{1}\left(F_{g e n}\right)$ for $n=2$. Let denote the number of irreducible components in $F_{c}$ by $r\left(F_{c}\right)$, and let $\operatorname{Sing}_{c}$ be Sing $\cap F_{c}$ : the affine singularities on $F_{c}$. Let $B_{x}^{4}$ be a small closed ball around a singular point $x$ of $F_{c}$. Then $H_{2}\left(F_{\text {gen }}, F_{c}^{\circ}\right)=\bigoplus_{x \in \operatorname{Sing}_{c}} H_{2}\left(F_{\text {gen }} \cap B_{x}^{4}, F_{\text {gen }} \cap\right.$ $\left.\partial B_{x}^{4}\right)=\bigoplus_{x \in \operatorname{Sing}_{c}} H_{2}\left(S^{2}\right)$, then the rank of $H_{2}\left(F_{g e n}, F_{c}^{\circ}\right)$ is the cardinal of $\operatorname{Sing}_{c}$, which is also the rank of Ker $\ell_{c}$ (by the exact sequence of the proof of proposition 2.2). Moreover $\chi\left(F_{c}\right)=\operatorname{rk} H_{1}\left(F_{c}^{\circ}\right)-r\left(F_{c}\right)-\# \operatorname{Sing}_{c}$.

$$
\begin{aligned}
\operatorname{rk} \operatorname{Ker}\left(h_{c}-\mathrm{id}\right) & =\operatorname{rk} \operatorname{Im} \ell_{c} \\
& =\operatorname{rk} H_{1}\left(F_{c}^{\circ}\right)-\operatorname{rk} \operatorname{Ker} \ell_{c} \\
& =r\left(F_{c}\right)-\chi\left(F_{c}\right)+\# \operatorname{Sing}_{c}-\# \operatorname{Sing}_{c} \\
& =r\left(F_{c}\right)-\chi\left(F_{c}\right) .
\end{aligned}
$$

Remark. We obtain the following fact (see [6]): if the fiber $F_{c}(c \in \mathcal{B})$ is irreducible then $h_{c} \neq$ id. The proof is as follows: if $r\left(F_{c}\right)=1$ and $h_{c}=$ id then from one hand $\operatorname{rk} \operatorname{Ker}\left(h_{c}-\mathrm{id}\right)=\operatorname{rk} H_{1}\left(F_{g e n}\right)=1-\chi\left(F_{g e n}\right)$ and from the other hand $\operatorname{rk} \operatorname{Ker}\left(h_{c}-\mathrm{id}\right)=$ $1-\chi\left(F_{c}\right)$; thus $\chi\left(F_{c}\right)=\chi\left(F_{\text {gen }}\right)$ which is absurd for $c$ in $\mathcal{B}$ by Suzuki formula, see [4].

\subsection{Vanishing cycles}

Now and until the end of this paper homology is homology with complex coefficients.

Vanishing cycles for eigenvalues $\lambda \neq 1$. Let $E_{\lambda}$ be the space $E_{\lambda}=\operatorname{Ker}\left(h_{c}-\lambda \text { id }\right)^{p}$ for a large integer $p$.

Lemma 2.3. If $\lambda \neq 1$ then $E_{\lambda} \subset V_{q}(c)$.

Proof. If $\sigma \in H_{q}\left(F_{g e n}\right)$ then $h_{c}(\sigma)-\sigma \in V_{q}(c)$. This is just the fact that the cycle $h_{c}(\sigma)-\sigma$ corresponds to the boundary of a "tube" defined by the action of the geometrical monodromy. We remark that this fact can be generalized for $j \geqslant 1$ to

$$
h_{c}^{j}(\sigma)-\sigma \in V_{q}(c) .
$$

Let $p$ be an integer that defines $E_{\lambda}$, then for $\sigma \in E_{\lambda}$ :

$$
\begin{aligned}
0 & =\left(h_{c}-\lambda \mathrm{id}\right)^{p}(\sigma)=\sum_{j=0}^{p}\left(\begin{array}{l}
p \\
j
\end{array}\right)(-\lambda)^{p-j} h_{c}^{j}(\sigma) \\
& =\sum_{j=0}^{p}\left(\begin{array}{c}
p \\
j
\end{array}\right)(-\lambda)^{p-j}\left(h_{c}^{j}(\sigma)-\sigma\right)+\sum_{j=0}^{p}\left(\begin{array}{c}
p \\
j
\end{array}\right)(-\lambda)^{p-j} \sigma \\
& =\sum_{j=0}^{p}\left(\begin{array}{l}
p \\
j
\end{array}\right)(-\lambda)^{p-j}\left(h_{c}^{j}(\sigma)-\sigma\right)+(1-\lambda)^{p} \sigma .
\end{aligned}
$$


Each $h_{c}^{j}(\sigma)-\sigma$ is in $V_{q}(c)$, and a sum of such elements is also in $V_{q}(c)$, then $(1-\lambda)^{p} \sigma \in$ $V_{q}(c)$. As $\lambda \neq 1, \sigma \in V_{q}(c)$.

Vanishing cycles for the eigenvalue $\lambda=1$. Let recall that vanishing cycles $V_{q}(c)=\operatorname{Ker} k_{c}$ for the value $c$, are cycles that "disappear" when the generic fiber tends to the fiber $F_{c}$. Hence cycles that will not vanish are cycles that already exist in $F_{c}$. From lemma 2.3, a cycle of $E_{\lambda}$ with eigenvalue $\lambda \neq 1$ is a vanishing cycle. We now study what happens for cycles associated to the eigenvalue 1 .

Let $\left(\tau_{1}, \ldots, \tau_{p}\right)$ be a family of $H_{q}\left(F_{g e n}\right)$ such that the matrix of $h_{c}$ in this family is:

$$
\left(\begin{array}{ccccc}
1 & 1 & & & (0) \\
& 1 & 1 & & \\
& & 1 & \ddots & \\
& (0) & & \ddots & 1 \\
& & & & 1
\end{array}\right) .
$$

Then, the cycles $\tau_{1}, \ldots, \tau_{p-1}$ are vanishing cycles. It is a simple consequence of the fact that $h_{c}(\sigma)-\sigma \in V_{q}(c)$, because for $i=1, \ldots, p-1$, we have $h_{c}\left(\tau_{i+1}\right)-\tau_{i+1}=\tau_{i}$, and then $\tau_{i}$ is a vanishing cycle. It remains the study of the cycle $\tau_{p}$ and the particular case of Jordan blocks (1) of size $1 \times 1$. We will start with the second part.

Vanishing and invariant cycles. Let $K_{q}(c)$ be the space of invariant and vanishing cycles for the value $c: K_{q}(c)=\operatorname{Ker}\left(h_{c}-\mathrm{id}\right) \cap V_{q}(c)$. Let us remark that the space $K_{q}(c) \oplus \bigoplus_{c^{\prime} \neq c} V_{q}\left(c^{\prime}\right)$ is not equal to $\operatorname{Ker}\left(h_{c}-\mathrm{id}\right)$. But equality holds in cohomology, see $[7]$.

Lemma 2.4. $K_{q}(c)=\ell_{c}\left(\operatorname{Ker} j_{c}^{\circ}\right)$.

This lemma just follows from the description of invariant cycles (proposition 2.2) and from the diagram of lemma 2.1. For $n=2$ we can calculate the dimension of $K_{1}(c)$.

Proposition 2.5. For $n=2$, $\operatorname{rk} K_{1}(c)=r\left(F_{c}\right)-1+\operatorname{rk} H_{1}\left(\bar{G}_{c}\right)$.

Proof. The proof will be clear after the following remarks:

(i) $K_{1}(c)=\ell_{c}\left(\operatorname{Ker} j_{c}^{\circ}\right)$, by lemma 2.4 .

(ii) $j_{c}^{\circ}=j_{c} \circ i_{c}$ with $i_{c}: H_{1}\left(F_{c}^{\circ}\right) \longrightarrow H_{1}\left(F_{c}\right)$ the morphism induced by inclusion. It is consequence of the commutative diagram:

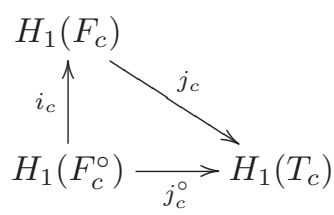


(iii) $\operatorname{rk} \operatorname{Ker} j_{c}^{\circ}=\operatorname{rk} \operatorname{Ker} i_{c}+\operatorname{rk}\left(\operatorname{Ker} j_{c} \cap \operatorname{Im} i_{c}\right)$, which is a general formula for the kernel of the composition of morphisms.

(iv) $\operatorname{Ker} j_{c} \cap \operatorname{Im} i_{c}=\operatorname{Ker} j_{c}$; we have the composition of maps:

$$
H_{1}\left(F_{c}^{\circ}\right) \stackrel{i_{c} \oplus 0}{\longrightarrow}\left(\operatorname{Im} i_{c} \oplus H_{1}\left(G_{c}\right)\right)=H_{1}\left(F_{c}\right) \stackrel{j_{c}}{\longrightarrow} H_{1}\left(T_{c}\right),
$$

and the restriction of $j_{c}, j_{c}: H_{1}\left(G_{c}\right) \longrightarrow H_{1}\left(T_{c}\right)$ is injective, so $\operatorname{Ker} j_{c} \subset \operatorname{Im} i_{c}$.

(v) $\operatorname{rkKer} i_{c}=\sum_{z \in \operatorname{Sing}_{c}} r\left(F_{c, z}\right)$, where $F_{c, z}$ denotes the germ of the curve $F_{c}$ at $z$.

(vi) $\operatorname{rk} \operatorname{Ker} j_{c}=\operatorname{rk} \operatorname{Ker} j_{\infty}=\sum_{P \in \operatorname{Dic}_{c}}\left(n\left(F_{P}\right)-1\right)=n\left(F_{c}\right)+\operatorname{rk} H_{1}\left(\bar{G}_{c}\right)-\operatorname{rk}\left(G_{c}\right)$, it has been proved in lemma 1.3.

(vii) $r\left(F_{c}\right)+\operatorname{rk} H_{1}\left(G_{c}\right)=n\left(F_{c}\right)+\sum_{z \in \operatorname{Sing}_{c}}\left(r\left(F_{c, z}\right)-1\right)$. This a general formula for the graph $G_{c}$, the number of vertices of $G_{c}$ is $r\left(F_{c}\right)$, the number of connected components is $n\left(F_{c}\right)$, the number of loops is rk $H_{1}\left(G_{c}\right)$ and the number of edges for a vertex that correspond to an irreducible component $F_{\text {irr }}$ of $F_{c}$ is: $\sum_{z \in F_{i r r}}\left(r\left(F_{i r r, z}\right)-1\right)$.

(viii) $\operatorname{rk} K_{1}(c)=\operatorname{rk} \operatorname{Ker} j_{c}^{\circ}-\# \operatorname{Sing}_{c}$ because $\operatorname{Ker} i_{c}$ is a subspace of $\operatorname{Ker} \ell_{c}$ so $\operatorname{rk} K_{1}(c)=\operatorname{rkKer} j_{c}^{\circ}-\operatorname{rkKer} \ell_{c}$ and the dimension of Ker $\ell_{c}$ is \# $\operatorname{Sing}_{c}$ (see paragraph 2.2).

We complete the proof:

$$
\begin{aligned}
& \operatorname{rk} K_{1}(c)=\operatorname{rk} \ell_{c}\left(\operatorname{Ker} j_{c}^{\circ}\right) \\
& =\operatorname{rk} \operatorname{Ker} j_{c}^{\circ}-\operatorname{rk} \operatorname{Ker} \ell_{c} \\
& =\operatorname{rk} \operatorname{Ker} j_{c} \circ i_{c}-\# \operatorname{Sing}_{c} \\
& =\operatorname{rk} \operatorname{Ker} i_{c}+\operatorname{rk} \operatorname{Ker} j_{c} \cap \operatorname{Im} i_{c}-\# \operatorname{Sing}_{c} \\
& =\operatorname{rk} \operatorname{Ker} i_{c}-\# \operatorname{Sing}_{c}+\operatorname{rk} \operatorname{Ker} j_{c} \\
& \text { by (viii) } \\
& \text { by (ii) and (viii) } \\
& \text { by (iii) } \\
& \text { by (iv) } \\
& =\sum_{z \in \operatorname{Sing}_{c}}\left(r\left(F_{c, z}\right)-1\right)+n\left(F_{c}\right)+\operatorname{rk} H_{1}\left(\bar{G}_{c}\right)-\operatorname{rk}\left(G_{c}\right) \quad \text { by (v) and (vi) } \\
& =r\left(F_{c}\right)-1+\operatorname{rk} H_{1}\left(\bar{G}_{c}\right) \text {. } \\
& \text { by (vii) }
\end{aligned}
$$

Filtration. Let $\phi$ be the map provided by the total resolution of $f$. The divisor $\phi^{-1}(c)$ is denoted by $D=\sum_{i} m_{i} D_{i}$ where $m_{i}$ stands for the multiplicity of $D_{i}$. We associate to $D_{i}$ a part of the generic fiber denoted by $F_{i}$. We briefly recall this construction (see [6]), let $V=\phi^{-1}\left(D_{\varepsilon}^{2}(c)\right.$ ) be a tubular neighborhood of $D$, we will identify the generic fiber $F_{\text {gen }}$ with $\phi^{-1}(s) \backslash \pi^{-1}\left(L_{\infty}\right)$ for a generic value $s \in \partial D_{\varepsilon}^{2}(c), \pi$ is the blow-up associated to $\phi$. There is a natural deformation retraction $R: V \longrightarrow D$, 
and we set $F_{i}=R^{-1}\left(D_{i}\right) \cap F_{\text {gen }}$. The filtration of the homology of the generic fiber is the sequence of inclusions:

$$
W_{-1} \subset W_{0} \subset W_{1} \subset W_{2}=H_{1}\left(F_{\text {gen }}\right),
$$

with

- $W_{-1}$ : the boundary cycles, that is to say, if $\bar{F}_{g e n}$ is the compactification of $F_{g e n}$ and $\iota_{*}: H_{1}\left(F_{\text {gen }}\right) \longrightarrow H_{1}\left(\bar{F}_{\text {gen }}\right)$ is induced by inclusion then $W_{-1}=\operatorname{Ker} \iota_{*}$;

- $W_{0}$ : these are gluing cycles: the homology group on the components of $F_{i} \cap F_{j}$ $(i \neq j)$;

- $W_{1}$ : the direct sum of the $H_{1}\left(F_{i}\right)$;

- $W_{2}=H_{1}\left(F_{g e n}\right)$.

The subspaces $W_{0}$ and $W_{1}$ depend on the value $c$.

Jordan blocks for $\boldsymbol{n}=\mathbf{2}$. For polynomials in two variables, the size of Jordan blocks for the monodromy $h_{c}$ is less or equal to 2. Let denote by $\sigma$ and $\tau$ cycles of $H_{1}\left(F_{g e n}\right)$ such that $h(\sigma)=\sigma$ and $h(\tau)=\sigma+\tau$. The matrix of $h_{c}$ for the family $(\sigma, \tau)$ is $\left(\begin{array}{ll}1 & 1 \\ 0 & 1\end{array}\right)$. We already know that the cycle $\sigma$ vanishes.

A large cycle is a cycle of $W_{2}=H_{1}\left(F_{\text {gen }}\right)$ that has a non-trivial class in $W_{2} / W_{1}$. According to [6] $\tau$ is large cycle; moreover large cycles associated to the eigenvalue 1 are the embedding of $H_{1}\left(\bar{G}_{c}\right)$ in $H_{1}\left(F_{\text {gen }}\right)$. So large cycles are not vanishing cycles. We have $\mathrm{rk} W_{2} / W_{1}$ equal to $\mathrm{rk} H_{1}\left(\bar{G}_{c}\right)$, this is also the number of Jordan 2-blocks for the eigenvalue 1 .

Vanishing cycles. We are now able to describe vanishing cycles. For all the spaces $W_{-1}, W_{0} / W_{-1}, W_{1} / W_{0}$ and $W_{2} / W_{1}$ the cycles associated to eigenvalues different from 1 are vanishing cycles (lemma 2.3).

Proposition 2.6. We have

$$
\operatorname{Ker}\left(h_{c}-\mathrm{id}\right)^{2} \cap V_{1}(c)=\operatorname{Ker}\left(h_{c}-\mathrm{id}\right) \cap V_{1}(c)=: K_{1}(c)
$$

and vanishing cycles for the eigenvalue 1 are dispatch as follows:

- for $W_{-1}: r\left(F_{c}\right)-1$ cycles (i. e. $\operatorname{rk} W_{-1} \cap K_{1}(c)=r\left(F_{c}\right)-1$ ),

- for $W_{0}$ : $\operatorname{rk} H_{1}\left(\bar{G}_{c}\right)$ other cycles (i. e. $\operatorname{rk}\left(W_{0} \cap K_{1}(c)\right) /\left(W_{-1} \cap K_{1}(c)\right)=$ $\operatorname{rk} H_{1}\left(\bar{G}_{c}\right)$ ),

- for $W_{1}$, no other cycle (i. e. $\left.\operatorname{rk}\left(W_{1} \cap K_{1}(c)\right) /\left(W_{0} \cap K_{1}(c)\right)=0\right)$,

- for $W_{2}$, no other cycle (i. e. $\left.\operatorname{rk}\left(W_{2} \cap K_{1}(c)\right) /\left(W_{1} \cap K_{1}(c)\right)=0\right)$. 
Proof. We have already remarked that large cycles (like $\tau$ ) associated to $\left(\begin{array}{ll}1 & 1 \\ 0 & 1\end{array}\right)$ are not vanishing cycles, so vanishing cycles in $W_{2}$ are in $W_{1}$, that is to say $W_{2} \cap \operatorname{Ker}\left(h_{c}-\mathrm{id}\right)^{2} \cap$ $V_{1}(c)=W_{1} \cap \operatorname{Ker}\left(h_{c}-\mathrm{id}\right)^{2} \cap V_{1}(c)$. Then the other vanishing cycles for the eigenvalue 1 are invariant cycles by $h_{c}$, in other words we proved that $\operatorname{Ker}\left(h_{c}-\mathrm{id}\right)^{2} \cap V_{1}(c)=K_{1}(c)$. We have $W_{1} \cap K_{1}(c)=W_{0} \cap K_{1}(c)$ because invariant cycles for $W_{1}$ that are not in $W_{0}$ correspond to the genus of the smooth part $F_{c}^{\circ}$ of $F_{c}$ (this is due to the equality $\left.\operatorname{Ker}\left(h_{c}-\mathrm{id}\right)=\ell_{c}\left(H_{1}\left(F_{c}^{\circ}\right)\right)\right)$. As they already appear in $F_{c}$, these cycles are not vanishing cycles for the value $c$. Moreover there are $\operatorname{rk} H_{1}\left(\bar{G}_{c}\right)$ Jordan 2-blocks for the eigenvalue 1 that provide $\operatorname{rk} H_{1}\left(\bar{G}_{c}\right)$ vanishing cycles (like $\left.\sigma\right)$ in $W_{0} / W_{-1}$. Finally, proposition 2.5 proves that $\mathrm{rk} W_{-1} \cap K_{1}(c)=r\left(F_{c}\right)-1$.

\section{References}

[1] E. Artal-Bartolo, P. Cassou-Noguès, and A. Dimca, Sur la topologie des polynômes complexes, Singularities (Oberwolfach, 1996), Progr. Math., vol. 162, Birkhäuser, Basel, 1998, pp. 317-343.

[2] E. Artal-Bartolo, P. Cassou-Noguès, and I. Luengo Velasco, On polynomials whose fibers are irreducible with no critical points, Math. Ann. 299 (1994), 477-490.

[3] A. Dimca and A. Némethi, On the monodromy of complex polynomials, Duke Math. J. 108 (2001), 199-209.

[4] Hà H. V. and Lê D. T., Sur la topologie des polynômes complexes, Acta Math. Vietnam. 9 (1984), 21-32.

[5] Lê D. T. and C. Weber, A geometrical approach to the Jacobian conjecture for $n=2$, Kodai Math. J. 17 (1994), 374-381, Workshop on Geometry and Topology (Hanoi, 1993).

[6] F. Michel and C. Weber, On the monodromies of a polynomial map from $\mathbb{C}^{2}$ to $\mathbb{C}$, Topology 40 (2001), 1217-1240.

[7] W. D. Neumann and P. Norbury, Vanishing cycles and monodromy of complex polynomials, Duke Math. J. 101 (2000), 487-497.

[8] _ Unfolding polynomial maps at infinity, Math. Ann. 318 (2000), 149-180. 